\title{
New ways with the solar nebula
}

\section{Was light from the primaeval Sun the agent of the differentiation of the planets as we know them? The density was probably too great for ordinary radiation pressure to suffice, but there are ways around that difficulty.}

WHETHER or not the general opinion of the origin of the Solar System is more or less correct, the simple view that everything began in a contracting nebula of roughly homogeneous chemical composition fails to carry quite the conviction of a well polished model. There are too many loose ends, such as the time-course of the evolution of the nebula in the early stages, before the Solar System came to resemble what we see today. With time, the loose ends will no doubt be tied together.

But there are also anomalies, departures from the expectations suggested by the simple model, which are more worrying, for just the reasons that a few grains of sand in an otherwise efficient lubricant may destroy the engine of a motorcar. The high mean density of Mercury has always been a bother. The circumstance that the Earth, Mars and Venus, similarly placed and roughly of the same mass, should have such very different atmospheres demands an explanation requiring a special assumption for each planet (such as life on the Earth, for example)

Part of the trouble is that knowledge of the Solar System has been accumulating too quickly for theorists' comfort. A few decades ago a then colleague, an ingenious, voluble and eccentric Ulsterman called W. H. ("Bill", as in Clinton) Ramsey, was able to fit on a smooth curve the known characteristics of the planets - mass, density and hardly anything else - by starting with the assumption that each had begun as a blob of solar nebula which had then been differentiated under the influence of the Sun. By the time he died (too young) in the late 1950s, Ramsey was already being overwhelmed by inconvenient facts.

Those who would make sense of the variations between the properties of the planets in the Solar System by starting with an undifferentiated solar nebula have a much more difficult task than Ramsey's: there is so much more to account for. The history of the Sun can be reasonably well inferred from what the stellar modellers have done, of course.

The Sun would have begun to form from the nebula by its collapse under its own weight, and its central regions would have been progressively heated by the energy thus liberated. So the outer regions of the collapsing nebula would have been exposed to an increasing outward flux of radiation of increasingly high frequency, infrared radiation first and radiation not very different from what we now see when nuclear reactions were switched on in the Sun's core.

Even so, it is far from clear whether the NATURE · VOL 361 · 18 FEBRUARY 1993 material from which the planets eventually formed was prevented from becoming part of the Sun by its inertia (which would be a function of the speed of collapse) or whether it was actively kept out by, say, radiation pressure (to use that term loosely). For stellar modellers, that is a question for a second or third approximation in understanding the process of star formation, but for those who would understand the history of the planets, it is a central question. Would there have been enough of the nebula left over to form them, for example, especially when the formation of the inner planets, rich in heavy elements as they are, would have been exceedingly wasteful of nebular material?

It is also clear that the process of differentiation and segregation in the remnant of the nebula must have continued long after the Sun became a star. To a first approximation, the preferential loss of light elements from the inner Solar System may be accounted for simply by the temperature on the surfaces of the inner planets (which is what Ramsey used to do).

But there are snags, one of which is the variation of the ratio of deuterium to hydrogen from the inner to the outer planets. These data, which have become accurate only because of planetary spacecraft, are remarkable. On Venus, the ratio (by atoms) of D to $\mathrm{H}$ is 1.6 per cent, roughly 100 times greater than on the Earth, but in the outer planets it is not very different from the cosmic value, which is a further order of magnitude less.

Two Russians, S. N. Atutov and A. M. Shalagin, appear to have provided a way out of this conundrum. Their conclusion is that the variations of the $\mathrm{D} / \mathrm{H}$ ratio cannot be explained by the difference of the velocities of hydrogen and deuterium atoms in the upper atmospheres of the inner planets, even though that effect is in the right direction. (The Maxwell distribution of velocities at a given temperature scales inversely with the square root of the mass, so that hydrogen is lost more easily than deuterium).

Nor, sadly, will radiation pressure in the ordinary sense suffice. Although atoms are impelled away from a source of radiation by the momentum of photons they may absorb, so that atoms that preferentially absorb radiation from a source are preferentially driven away from it, that mechanism is effective only when the density is small enough for collisions to be rare. By definition, that cannot be have been the case in the early remnant of the solar nebula. So they fall back on a suggestion, put forward in the 1970s by Gel'mukhanov and Shalagin, that a different process, which they call "light-induced drift". may do the trick instead.

In case that sounds like special pleading, a group from the University of Leiden has now, in a neat experiment, shown that even in relatively dense gases, molecular differentiation is possible (Phys. Rev. Lett. 70, 742; 8 February 1993). Evidently H. I. Bloemink, J. M. Boom-Engering, E. R. Eliel and L. J. F. Hermans have it in mind that the segregation of hydrogen and deuterium takes place at a very early stage in the condensation of the Sun, when the temperature of the outer nebula is $800 \mathrm{kK}$ or so, and molecules rather than atoms would predominate.

For light-induced drift to be effective, it is essential that the chance that a photon will be absorbed should depend on the motion of a molecule, towards or away from the source of radiation, and that absorption should then bias the kinematics of the molecules towards drift. Bloemink et al. have worked with water molecules (certainly constituents of the protonebula), irradiating them by a laser tuned to the frequency of the principal vibration band of the molecule, yet slightly to the red side of it while still within the Doppler-broadened width.

The guiding principle is that photons should be absorbed only by molecules moving towards the radiation source. The expectation that they would then, by virtue of their vibration, be more likely to collide with others and be randomized in velocity, leads directly to the idea that there should be a bias in their overall direction away from the source. The awesome thing is that exactly this phenomenon has been confirmed by irradiating capillary tubes $30 \mathrm{~cm}$ long containing water molecules and buffer gas (helium, for example). Water molecule concentrations have been increased by 2 or 3 parts of a million down the laser beam.

All that is a technical triumph, but two questions arise for the Solar System modellers. How can such a mechanism separate deuterium from hydrogen? Because the water molecule absorption bands are stronger than those of, say, HDO. And where in astrophysics is there a source of radiation fine-tuned as an expensive laser to be just to the red of the principal vibrational absorption band of $\mathrm{H}_{2} \mathrm{O}$ ? Why not the red-shifted output of vibrational photons from water molecules on the surface of the massive proto-Sun?

Bloemink et al. say that the numbers fit, and that light-induced drift would suffice to have cleaned the inner Solar System out to Venus of $\mathrm{H}_{2} \mathrm{O}$ in about 10 million years. It sounds good.

John Maddox 